\title{
Neoclassical Theory of X-Ray Scattering by Electrons
}

\author{
V.V. Aristov \\ Institute of Microelectronics Technology and High Purity Materials RAS, \\ Russia
}

\section{Introduction}

Many successes in physics of 20th century associated with quantum mechanics in the statement, which was given by the N. Bohr, W. Heisenberg, M. Born, W. Pauli and others. It is known as "Copenhagen interpretation". It bases on the statement that the purpose of quantum theory is the description of results of observation, instead of getting knowledge about reality and the processes occurring in material systems in an interval between the first observation and the next. Moreover: "Any attempt to find such a description would lead to contradictions" (Heisenberg, 1958). Canonization of this opinion considerably changed a view at the purpose of researches, led to the confidence accepted today by the majority of physicists that "the quantum mechanics has rejected usual classical mechanics determinism in behavior of microobjects", and that "the aim of quantum mechanics to give a method for finding probability distributions for various physical values in various states of microobjects" (Akhiezer \& Polovin, 1973). Postulation a fundamental nature of quantum mechanics "obscurity" has led to the statement on completeness of quantum mechanics, i.e. that there are no parameters (probably hidden) which define physical sense of processes in quantum system and give fuller description of the nature, than are based on probability functions or a density matrix.

This radical change of the research concept was denied by many of those who stood at the basis of the quantum theory - M. Planck, A. Einstein, E. Schrödinger, M. von Laue, and L. de Broglie. In second half of 20th century, dispute over reality and search of physical reasons, causing the quantum phenomena, has led to various formulations of quantum mechanics the theories containing hidden variables, describing behavior and evolution of quantum systems in space and time. Herein D. Bohm mechanics, based on de Broglie idea about a "pilot wave" directing electron movement (Bohm, 1952), and the semiclassical or neoclassical electrodynamics theory developed by E. Jaynes and his colleagues (Crisp \& Jaynes, 1969), based on Schrödinger idea (supported by E. Fermi) that square of wave function describes not probability, but actual charge density distribution in atom (Schrödinger, 1926). In the same years J. Bell demonstrated, that contrary to belief of the majority of physicists about impossibility of hidden variables existence, the nonlocality of quantum mechanics reflects nonlocality of hidden variables (Bell, 2004). Nevertheless, today, in spite of enough numerous researches directed on revision of view of Copenhagen interpretation founders, the disputes round the purposes of physical researches and the 
essence of hypotheses and postulates, containing in the theory, majority of physicists are perceived as the philosophical debates representing only historical interest. "...truth does not triumph by convincing its opponents and making them see the light, but rather because its opponents eventually die..." (M. Planck). Such point of view is grounded usually by impossibility to offer any significant experiment distinguishing this realistic theory from canonical quantum mechanics. This opinion is deeply erroneous. Quantum mechanics from the very beginning developed as an empirical science. Its formulations, models were formed by only trial and error method. Any discrepancies of quantum models with experimental results were removed by imposing of "exclusion principle" for any processes, which should be observed according to the logic of classical physics, were removed by acceptance of postulates on principles of matter behavior in quantum world and hypotheses about a structure and properties of quanta. As a result of such "development" was creation of purely mathematical theory with the logic which is initially not reduced to the logic of classical physics. Such theory necessarily should meet difficulties in definition of physical sense some the phenomenon: "Quantum electrodynamics is not a perfectly consistent theory" (Dirac, 1965). "It is plagued by divergences, some of which are carried over from the classical theory of electromagnetic fields, and some of which are introduced by the procedure of quantizing the electromagnetic field. Quantizing a field that has an infinite number of degrees of freedom seems to lead unavoidably to an infinite amount of energy in the zero-point oscillations. Furthermore, the usual QED derivation of spontaneous decay, the Lamb shift, and the calculation of the anomalous moment of the electron seem to require these zero-point oscillations. Nevertheless, it is difficult to rationalize that these zero-point oscillations actually exist in nature" (Crisp, 1990).

Other fundamental difficulty of quantum mechanics in explaining of an interference of particles - photons, electrons etc. R. Feynman wrote about an interference problem on two slits: "Impossible, absolutely impossible to explain it any classical way ... has in the heart of QM. Really, it contains the only mystery" (Feynman, 1965).

Usually considering, the mysticism inherent in quantum description of some experiments is eliminated by replacement of the Copenhagen interpretation most popular in quantum physics by one of equivalent interpretations. It is considering, that all formulations (and them more than 10) despite "differ dramatically in mathematical and conceptual overview, yet each one makes identical predictions for all results" (Styer et al., 2002).

Perhaps, firm belief in correctness of quantum mechanics conclusions is connected with considering in quantum mechanics simple enough phenomena isolated from others. We will below analyze complex experiments in X-ray physics where the various effects are the consequence of the same process but for its explanation till now theory formulates assumptions, not agreed among themselves, and by that we will prove, all mysticism of behavior of quantum particles is connected only with the theory. Important, that a lot of troubles and "obscurities" inherent in quantum physics, are concentrated in physics of X-ray scattering.

In this chapter, results of experimental research of hard x-ray radiation scattering will be compared to those theories, which have arisen for their explanation. It is interesting to note, already in the early twenties of last century the common theory of scattering of electromagnetic radiation could be created. Instead of it, unique process of radiation 
scattering by atoms of matter began to be considered as consisting of the several independent phenomena described by various mechanisms of scattering. Theories describing the scattering are:

Classical (Thomson) theory: electromagnetic wave scattering by point electrons. All electrons of atom scatter incoherently with each other. Frequency of a scattering wave coincides with incident frequency. Possibility of existence of the "big" electron having the certain size and the form is supposed.

Quantum (Compton) theory: the photon interact with the point electron in atom in the state, defined at the moment of scattering in coordinate and momentum corresponding to the solution of Schrödinger wave equation. This scattering named as Compton or incoherent because frequency of scattering radiation (new photon frequency) depends on a scattering angle and an initial momentum of an electron which it has at the moment of collision with a primary photon.

Neoclassical theory of scattering (NCT) - base for X-ray diffraction theory and X-ray crystallographic analysis: electromagnetic wave scattering by electron distribution in atom. This distribution is set by Schrödinger function, which is understood not as function of probability distribution of point electron in atom but as the real charge density. It is coherent (Bragg and Rayleigh) scattering without frequency changing.

The listed theories - classical, quantum and NCT are applied not to the description of scattering of X-ray radiation by atomic electrons, but for the description of separate fragments of scattering spectrum in various models of an atom structure and radiation properties.

From here follows singlevalued, though also a paradoxical conclusion. In the modern physics, there is no theory of scattering of hard electromagnetic radiation not only by bound, but also by free electrons.

Situation with treatment of $x$-ray radiation scattering on matter reminds the story about an elephant and the group of blind men. They touch an elephant at different parts to learn what it is like. As a result each of them formed his own "the theory of an elephant". Disputes what theory more correct are useless. Everyone reflects only a part of the general essence. It is necessary to see "elephant" entirely. For this purpose, in the case of X-ray scattering phenomena, it is necessary to rethink available experimental data, recognizing, that all of them reflect uniform process of scattering, and all theories existing for today describing its separate fragments are not perfectly correct, and provide some misrepresentation about a matter structure.

Basic concept of quantum electrodynamics is the concept about a photon and electron as a point-like particle. The most convincing, but actually, the first and unique proof of photon existence is Compton effect: changing and angular dependence of the scattered (secondary) $x$-ray radiation wavelength in comparison to the incident radiation.

For Compton effect explaining by photons scattering it is necessary, that the electron in atom: 1) was point-like, 2) had a certain momentum - instant velocity and a direction of movement in atom (theory of impulse approximation), explaining broadening of Compton line. 
On the other hand, as noted above, X-ray diffraction method bases on the assumption that electromagnetic wave scatters not on separate point electrons, but on a charge distributed in atom. This mechanism of scattering is put in a basis of methods of atoms and molecules structures definition, research of crystal real structure. Clearly, that it cannot be realized from quantum mechanics point of view. Imagine, that the distance between slits (electrons in atoms) quickly changes in time under some probability law in a big area defined by Schrödinger function, then named Feynman "mystical" process of an interference of photons on two slits becomes inconceivable. Thus it is clear, in experiments on $x$-ray diffraction (i.e. at coherent scattering), X-rays, scattered by electrons of a crystals, is electromagnetic radiation, and the charge occupies certain volume in atoms, instead of consists of point electrons. However, if it so, what conclusion should be made about plausibility of quantum origin of Compton effect, and other effects arising at X-ray scattering?

For the answer to this question, at the analysis of results of X-ray radiation scattering experiments we will base on representations about mechanisms of scattering, that are used in the neoclassical theory. We will see, both effects of scattering, "coherent" (Rayleigh) and "incoherent" (Compton), represent in a spectrum only phenomenon: scattering of electromagnetic radiation on a volumetric electron charge which structure is deformed in the field of atomic forces. In such understanding of scattering process Thomson theory plays the role of the theory of wave scattering on the elementary charge contained in small volume $\mathrm{dV}$ of electronic density.

\section{Classical theory of scattering (1903-1923)}

To develop new theory on x-ray scattering we are used the technology suggested by J. Dodd at a classical treatment of Compton effect (Dodd, 1983): «I should like to include a little fantasy: to presented that we stand near the beginning of the $20^{\text {th }}$ century and attend to discover the lands for the interaction between light and matter using the classical theory of the day being guiding by experiments which in principle could be performed near that time».

\subsection{Basic conceptions and models of scattering used at first two decades in $20^{\text {th }}$ century}

The view of physics at the time we consider is dominated by theories of J. Maxwell (explanation of electromagnetic fields) and H. Lorentz (force equation explained how electrical charges and current interact). We have learned, that light propagation as a wave of electromagnetic fields described by these equation. Einstein's "lichtquanten" hypothesis did not obtain wide acceptance from the physicists at the time. Von Laue, for example, was opposed the light quanta and suggested that quantization resided in matter, not radiation (at least in x-ray wave region). Though, Einstein in 1918 wrote "I do not doubt any more, the reality of radiation quanta", in 1921 he complained to P. Ehrenfest that "problem of quanta was enough to drive him to the madhouse" (Kidd et al., 1989).

Discussions of size and shape of electron followed from point of view of different physical properties such as magnetic properties, possibility of electron to be compressed because 
external force to produce Lorentz radiation force for it compensation. The most popular model was electric charge compressed to shape of sphere of radius is equal to

$$
r_{e}=\frac{e^{2}}{m c^{2}}=2.82 \cdot 10^{-15} m
$$

Later Dirac suggests mathematic point like model of electron for quantum electrodynamics theory. Results of some experiments on the $\mathrm{X}$ and $\mathrm{Y}$ rays led to assumption that electron may be flexible ring or spherical shape distributed charge of electricity with radius order

$$
\Lambda_{e}=\frac{h}{m c}=2.42 \cdot 10^{-12} m
$$

(Bergman, 2004). From point of view of investigation of any real body structure and form, it is logical to use electromagnetic radiation with length of a wave, comparable with assumptive size of structure details of investigated object. Usual X-ray radiation satisfies to such criterion for atomic structure of matter: in X-ray experiments typical investigation radiation with wave length $0.5 \AA-2 \AA$ are used. From this point of view, for the theory of Xray scattering it was reasonable to start with assumption about point electron.

\subsection{Thomson theory - Scattering by point elementary charge}

In 1903 J. Thomson published the theory of scattering of electromagnetic radiation by point charge of an electricity. As noted above, the model of a point electron with radius $\mathrm{r}_{e}$ was offered along with model of an electron with the size of an order $\Lambda_{e}$

In this case, it is necessary, that "elementary charges", parts of the "big" electron, scatter coherently and independently, and relation of charge quantity to mass in volume $\mathrm{dV}$ in each part remains constant and equal to $e / m$, where $e$ and $m$ charge and mass of electron (Compton, 1919a). Assume charge distribution in a free electron ${ }^{1} \rho(r)=e|\psi(r)|^{2}$, mass $m|\psi(r)|^{2}$.

In the classical theory of scattering is assumed, that electron can have the sizes of an order of $2 \cdot 10^{-12} \mathrm{~m}$ and can consist of elementary charges in which the relation $\mathrm{e} / \mathrm{m}$ is constant. It means, that

$$
\frac{d e}{d m}=\frac{e}{m}, d e=e|\psi|^{2} d V, d m=m|\psi|^{2} d V, \int|\psi|^{2} d V=1
$$

On Fig. 1 the scheme of scattering plane electromagnetic wave with wavelength $\lambda_{0}$ and frequency $v_{0}$ on an electron consisting of elementary charges. Any "elementary" volume $\mathrm{dV}<<(\mathrm{h} / \mathrm{mc})^{3}$ inside distribution of $\rho(r)$ looks as elementary electronic charge with constant charge to mass relation.

${ }^{1}$ In $\mathrm{QM}$, for free electron $\psi(\mathrm{r})=\delta(\mathrm{r})$. For the electron in atom probability of its position in any point are calculated and presented by Schrödinger function $|\psi(\mathrm{r})|^{2}$. Acceptable distribution of electron density in NCT is under discussion in the paragraph 3. 


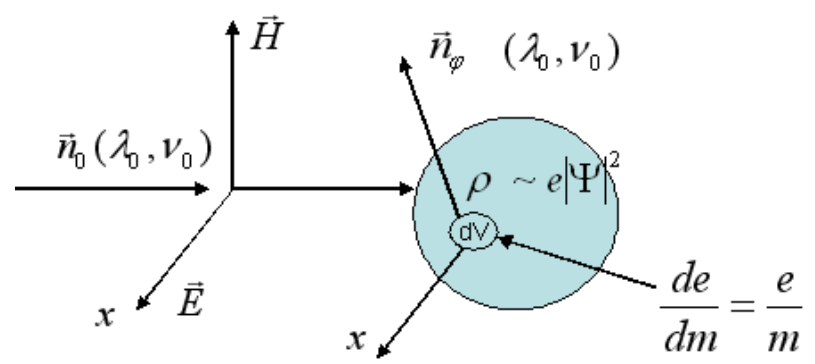

Fig. 1. To the theory of electromagnetic wave scattering by electron. Charge electron density $\rho$ is presented by distribution described by function $|\psi|^{2}$.

Each charge oscillates in a direction of electric field $\vec{E}$ under the influence of force:

$$
F=-d e \frac{1}{c} \frac{\partial A_{0}}{\partial t}
$$

where

$$
\frac{1}{c} \frac{\partial \vec{A}_{0}}{\partial t}=-\vec{E}_{0}
$$

$\vec{A}$ - vector potential of a field. The movement equation of an elementary charge is:

$$
\ddot{x}=\frac{e}{m} \vec{E}_{0}(t) \text { or } \vartheta(t)=-\frac{e}{m c} \vec{A}_{0}(t)
$$

Here $\vartheta(t)$ - velocity of fluctuations of an electron. Thus along an electron in direction $\vec{n}_{0}$ of distribution of an electromagnetic field current $J(r, t)$ flows. Thus:

$$
J(r, t)=e|\psi(r)|^{2} \vartheta(t)
$$

Further we consider only spherical symmetric functions $\psi(r)$ for which it is convenient to use polar co-ordinates - radius vector $\bar{r}$ and polar angles: $\varphi$ - an angle between vectors $\vec{n}_{0}$ and - $\vec{n}_{\varphi}, \vec{n}_{\varphi}$ - a direction of propagation of a scattering wave, $a$ - an angle between vectors $\vec{E}$ and $\vec{n}_{\varphi}$, in this case $d V=d x d y d z=r^{2} \sin \alpha d r d \alpha d \varphi=r^{2} d r d \Omega$

Let's define parameters of scattering radiation for a point electron when $|\psi(r)|^{2}$ is $\delta$ function. This condition is satisfied at radiation wavelengths $\lambda>10^{-2} \AA$, most widely used in X-ray crystallographic analysis. Besides, the equation (3) is valid in small fields $\vec{A}$, with oscillation amplitude $x_{\max }<\Lambda_{e}$.

Easy to show, scattering of a polarised wave by a point charge leads to dipole waves, intensity equation

$$
d|A|^{2}=\left(\frac{e^{2}}{m c^{2}}\right)^{2}\left|A_{0}\right|^{2} \sin ^{2} \alpha d \Omega
$$


Then differential cross section $d \sigma_{0}$ and cross section $\sigma_{0}$ is:

$$
\frac{\mathrm{d} \sigma_{0}}{\mathrm{~d} \Omega}=\mathrm{r}_{\mathrm{e}}^{2} \sin ^{2} \alpha ; \quad \sigma_{0}=\frac{8}{3} \pi \mathrm{r}_{\mathrm{e}}^{2}
$$

Notice one essential circumstance. At making above assumptions of structure and properties of the "big" electron, the value of scattering cross section is proportional to $\mathrm{r}_{\mathrm{e}}$.

Therefore, for the "big" charge easy to write the equation for amplitude and intensity scattered in direction $n_{\varphi}$ in limit far field diffraction zone $\mathrm{R}>>\mathrm{r}_{\mathrm{e}}(R$ - distance from an electron to a point of observation). Assume big electron scattering function in a direction $n_{\varphi}$ :

$$
\begin{gathered}
f_{e}\left(k_{0}-k_{\varphi}\right)=\int|\psi(r)|^{2} e^{2 \pi i\left(k_{0}-k_{\phi}\right) r} d V \\
k_{0}-k_{\varphi}=\frac{n_{0}-n_{\varphi}}{\lambda_{0}}=\vec{H}, \quad|\vec{H}|=\frac{2 \sin \frac{\varphi}{2}}{\lambda_{0}}
\end{gathered}
$$

$\vec{H}$ - vector in space of reciprocal wave vectors.

In this case scattering cross section:

$$
\frac{d \sigma(\vec{H})}{d \Omega}=r_{0}^{2} \sin ^{2} \varphi \cdot\left|f_{e}(\vec{H})\right|^{2}, d \sigma(\vec{H})=d \sigma_{0}\left|f_{e}(\vec{H})\right|^{2}
$$

According to Rutherford atomic model each electron in atom scatter short-wave radiation independently (incoherently) from other electrons. Thomson model also suggest, that intensity of scattering by each atom is proportional to amount of atomic electrons Z:

$$
I=\sigma_{0} Z
$$

In his experimental researches, C. Barkla demonstrates that electrons actually scatter independently and incoherently (Barkla, 1911), at least, in atoms of light elements (the neat result of this researches was definition the number of electrons in atom). Barkla also discovered polarization of X-ray radiation and validity Maxwell theory for calculation of Xrays scattering, thus identified it with the electromagnetic waves, having very short length in comparison with a visible region. P. Evald took for the basis of the wave propagation theory in crystals and assumed that wavelengths of X-ray radiation are comparable with interplane distances. This hypothesis was the basis for Laue experiment (performed by W.Friedrich and P. Knipping in 1912) for discovering X-rays diffraction on crystals. A year later, the father and son Braggs created the theory of Bragg diffraction, and the device for spectral analysis of scattering radiation. Discovering of diffraction of X-ray radiation has allowed to draw a remarkable but paradoxical conclusion that, in our opinion, was a forerunner of the subsequent "obscurity" the quantum physics. In spite of the fact that the majority of experiments shows, that electrons in each atom scatter independently from each other, the lattice atoms in a crystal, under certain conditions (Bragg conditions), become coherent scatterers for $\mathrm{X}$-ray radiation. 


\subsection{Compton theory - Scattering by finite size electron}

Among other important experimental results it is necessary to mention one more result, that did not become discovery but should become. D. Florance showed, that the angular diagram of X-rays scattering $I(\varphi)$ is asymmetric. $I\left(0^{\circ}\right)>I\left(180^{\circ}\right)$, and dependence $I\left(180^{\circ}\right) / I\left(0^{\circ}\right)$ rapidly decreases with $\lambda_{0}$ decreasing (Florance, 1910).

These results allow to suppose that the electron has the size comparable to x-ray radiation wavelengths. These years Mie and Debay already created the theory of light scattering on dielectric and metallic small diameter spherical particles, with diameter comparable to radiation wavelength (Mie, 1908; Debye, 1909). Rigorous solution problem of diffraction plane wave on homogeneous sphere of any diameter and structure was obtained. The next years many authors analyzed various aspects of this problem. In present paragraph, consider such researches in $\mathrm{x}$-ray wavelength region.

In 1918-1919 Compton dedicate theoretical researches to analysis of experimental data of xray scattering on electrons (Compton, 1919a,b). Fig.2 represents experimental measurement (circles) of relative intensity radiation scattered at different angles when the hard X-ray $(\lambda$ $=0.09 \AA$ ) from radium bromide traverse a plate of iron (Florance, 1910), and calculated curves of angular scattering dependence of unpolarized wave (Compton, 1919a). In figure, calculated curves for "big" spherical shell electron with radius $r=2 \cdot 10^{-10} \mathrm{~cm}$ - internal continuous line; for an electron in the form of a ring (ring electron, $r=2 \cdot 10^{-10} \mathrm{~cm}$ ) - dashed line; for point charge - an external continuous line.

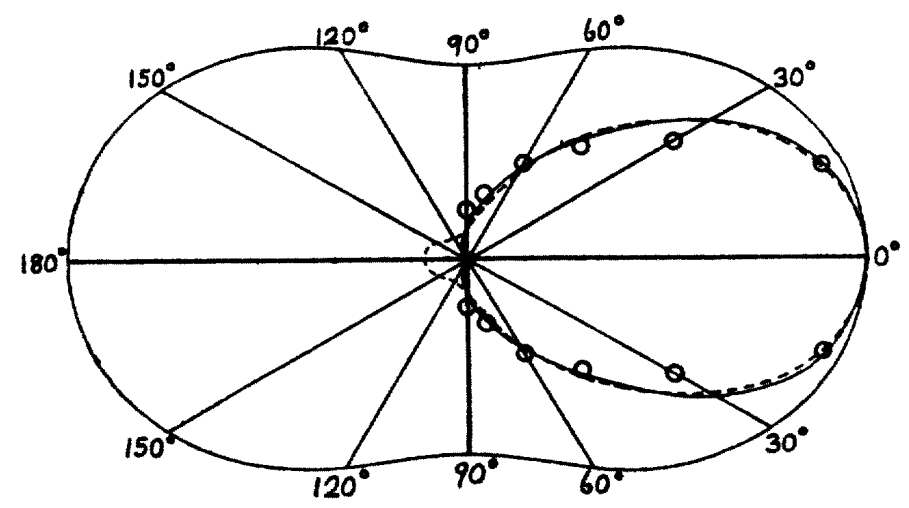

Fig. 2. Experimental (Florance, 1910) and theoretical (Compton, 1919a) angular dependencies integral scattering intensity from point-like and "big" electron with different charge distribution $\rho(r)$ (spherical shell and ring electron with radius $2 \cdot 10^{-12} \mathrm{~m}$ ), see text above.

Compton for the definition of distribution $\rho(r)$ has analyzed not only results of measurements of angular diagram of scattering. Analyzing measurements of dependence an absorption coefficient by various materials, he demonstrated, that $\mu_{a}$ - factor of atomic absorption of energy in an electron distributed in some volume, is defined by the sum consisting of fluorescent absorption, and scattering $\sigma$, depending on wavelength $\lambda_{0}$ : 


$$
\mu_{a}=k Z^{4} \lambda_{0}^{3} \Theta\left(r, \lambda_{0}\right)+\sigma\left(r, \lambda_{0}\right)
$$

$k=$ const, $r$-electron radius, functions $\Theta$ and $\sigma$ calculated for flexible ring electricity, are expressed approximately by the equations

$$
\Theta=14.8\left(\frac{r}{\lambda_{0}}\right)^{2}+93.6\left(\frac{r}{\lambda_{0}}\right)^{4}+\ldots, \sigma=\sigma_{0}\left(1-26.6\left(\frac{r}{\lambda_{0}}\right)^{2}+524\left(\frac{r}{\lambda_{0}}\right)^{4} \ldots\right)
$$

Formula (9) demonstrated, that experimental data well coincide with calculated at $\mathrm{r} \approx 0.8 \cdot 10^{-10} \mathrm{~cm}$.

Absorption measurements were less precise, than scattering measurements, but values of the calculated radiuses of an electron according to these various methods of measurements showed, that the electron is a particle with radius of an order 10-12 m. Such coincidence of experimental values of scattering and absorption, obtained at determination of electron radius, is especially important in view of dispute, continued more than centuries on character of interaction of radiation with matter. In conclusion review of first two decades results obtained by theory of scattering hard electromagnetic radiation, it is necessary to discuss in brief a problem of absorption and radiation by the charged particle of electromagnetic impulse and energy. This problem has been solved in classical electrodynamics more than 100 years ago. Compton in 1923 has offered the new decision, in fact reducing a problem of an exchange of energy and an impulse between field and electron to a mechanical problem of particles collision. His suggestion leads to dramatic consequences, especially for physics of $\mathrm{X}$-ray radiation scattering.

\subsection{Absorption of an impulse of an electromagnetic field, Lorentz forces - Impulse and energy conservation laws}

Consider scattering of an electromagnetic wave by an elementary charge. Force $F_{S}$, acting from a field on an electron, is equal to average value of an impulse absorbed in unit of time.

$$
F_{S}=\frac{8 \pi}{3} r_{e}^{2} \frac{E}{4 \pi}^{2} \vec{n}
$$

A charge particle on being accelerated by the force (10) recover electromagnetic energy and itself losses energy. H. Lorentz point to occurrence of braking force of an electron at radiation of X-ray electromagnetic wave (Lorentz, 1905). Loss of energy is interpreted by him as caused by a force $F_{L}$ acting on the particle given in value equal to $F_{S}$ and opposite to it:

$$
F_{L}=-F_{S}
$$

The sense of the equations (11) and (10) that the elementary charge in the electromagnetic field gets and loses energy and an impulse simultaneously. Therefore neither an electron at rest, nor a moving electron with constant velocity, does not change the kinetic energy at radiation scattering. Equality of forces $F_{L}$ and $F_{S}$ - is a consequence of equality of energy of the waves absorbed and reradiated by an electron at scattering by it of an electromagnetic wave. Presence of Lorentz force was checked experimentally though the physical sense of it origin remains till now not clear. In 1945 discussing this problem J. Wheeler and R. Feynman 
wrote: "The origin the force of radiative reaction has not been really so clear as its existence". Lorentz, explaining the mechanism radiative reaction forces found the cause in final size of electron and particle elasticity. Under the influence of external forces the sizes of a particle change, there are internal pressure, resistance to external forces. Dirac offered method of its calculation, come from point particle model and not trying to explain physical origin of radiation damping. On base of Dirac solution Wheeler and Feynman offered the scheme of creation braking forces in that the electron simultaneously radiates a spherical wave (out, retarded wave) and accepts another wave (in, advanced wave). In this case the semidifference of forces acting on an electron is equal to Lorentz force of any elementary charge (1). Therefore, equation (11) also as (7), is universal and correct at any form of an electron. The electron scatter the energy proportional to its cross section, and electromagnetic field loses the energy equal to the same value. The energy lost by a field comes back in the form of the sum dipole electromagnetic waves radiated by elementary charges of the big electron. The electron, absorbing energy, absorbs also proportional amount of an impulse that leads to occurrence of Lorentz force equal and opposite $\mathrm{F}_{\mathrm{S}}$.

Note fundamental meaning of a conclusion that follows from the above consideration. The equation (3) of charge transverse oscillation describes the mechanism of energy extraction from primary wave field and simultaneously returning back this energy by radiation dipole waves. The equation (11) confirms: absorption of an impulse of a field at energy absorption causes force acting on an electron in opposite direction at energy radiation.

\subsection{Classical theory: Short "Golden age" and swift break-down}

In conclusion of the section we will underline some important statements:

- X-rays are electromagnetic radiation, the hypothesis about radiation quanta was discussed, but did not find support in the scientific community up to 1923;

- Electron is a main reradiating matter. Cross section of scattering proportional to the size $r_{e}=\frac{e^{2}}{m c^{2}}$ named classical radius;

- The main part of radiation scattered by individual electrons in atom are incoherent;

- Electrons in the atoms from a crystal lattice is a coherent scatter under certain conditions (Bragg diffraction conditions);

- Hard X-ray radiation scattering experiments shows the possibility for electron to have a radius in order to $\mathrm{h} / \mathrm{mc}$.

These years were developed methods of the x-ray analysis of molecules and crystals structure, were outlined methods electronic structure analysis of atoms and electrons structure itself. Development of common theory of hard radiation scattering by electrons has been interrupted in 1923. Compton, and, independently, Debay, offered the theory explaining effect "softening" by scattering of particles of light (photons) on point electrons (Compton, 1923; Debye, 1923). "Softening" effect (found out earlier many authors (Eve, 1904; Barkla, 1904)) was: "back" scattering radiation (on large angles $\varphi$ ) absorbs more strongly, than "forward" scattering radiation (on small angles). In experiments Gray and Compton, executed independently, has been shown, that this effect is caused by scattering radiation wavelength increasing with increase in scattering angle $\varphi$ (Gray, 1920; Compton, 1922). This effect is known today as Compton effect. 
Fig. 3 presents Compton spectra of synchrotron radiation (Bessy-2) $\lambda_{0}=0.88 \AA$ scattered on carbon crystal.
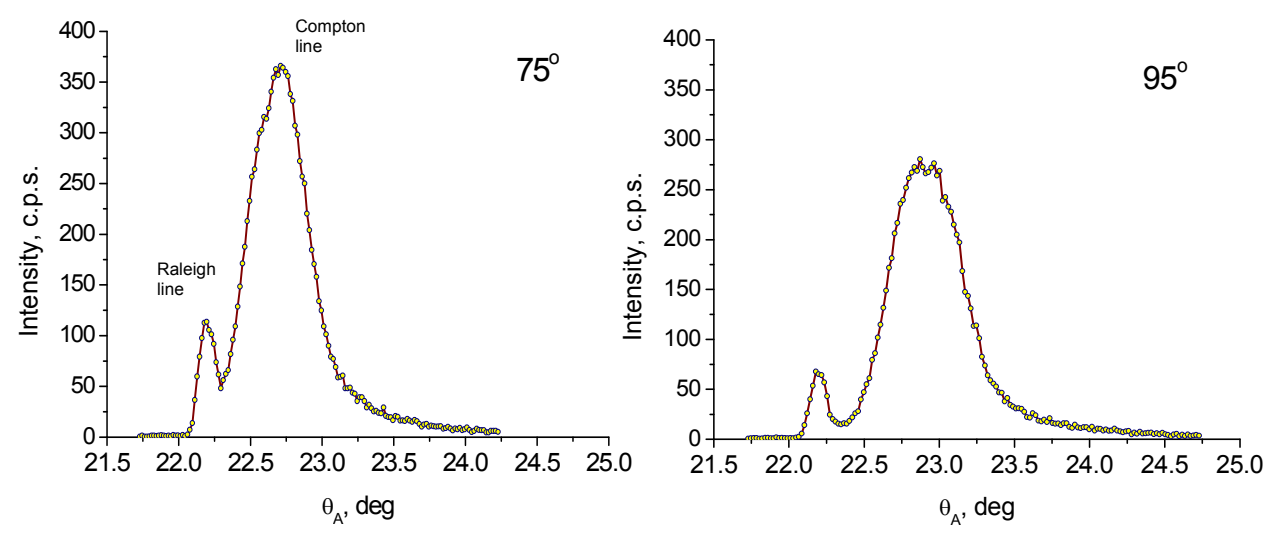

Fig. 3. Experimental scattering spectra $14 \mathrm{keV}$ on diamond at $\varphi=75^{\circ}, 95^{\circ}$. Center of Compton line at $\varphi=95^{\circ}$ approximately corresponds to $\Delta \lambda=\Lambda_{\mathrm{e}}$ (see equations (12)). $\theta_{\mathrm{A}}$ - angle of Ge crystal analyzer.

At scattering by electrons of carbon atoms, the spectrum contains a weak narrow line with wavelength which occurrence was expected according to the classical theory of scattering. It is surprising, that intensity of this unmodified line (named coherent or Rayleigh line), makes some percent from full intensity of scattering. This part of spectra is responsible for diffraction phenomena and widely used for the structural analysis, X-ray optics and interferometry. This line responsible also for formation of atomic scattering factor and refraction. Much more intensive part of scattering spectrum contains the wavelengths $\lambda>\lambda_{0}$. This is "Compton" part of scattering spectra, considered in quantum theory as incoherent. Experimentally, with very high accuracy, it has been established, that line centre (its wavelength and frequency) determinate by equations:

$$
\begin{gathered}
\lambda^{\prime}(\varphi)=\lambda_{0}+\Lambda_{e}(1-\cos \varphi) \quad v^{\prime}(\varphi)=\frac{v_{0}}{1-\frac{v_{0}}{v_{e}}(1-\cos \varphi)} \\
\frac{\Delta \lambda^{\prime}}{\lambda_{0}}=\frac{\Lambda_{e}}{\lambda_{0}}(1-\cos \varphi), \frac{\Delta v^{\prime}}{v^{\prime}}=-\frac{v_{0}}{v_{e}}(1-\cos \varphi) \\
\Lambda_{e}=\frac{h}{m c}, v_{e}=\frac{m c^{2}}{h}, \frac{v_{0}}{v_{e}}=\frac{\Lambda_{e}}{\lambda_{0}}
\end{gathered}
$$

The Compton line is very wide $\delta \lambda \approx \Delta \lambda^{\prime}$ and from this point of view it is partially coherent. Value of $\delta \lambda$ of "incoherent" Compton line is only 1-2 orders more than it is for coherent Rayleigh line. 
Main part of the radiation energy, scatter by an electron, is contained in a wide spectrum with wavelengths $\lambda^{\prime}>\lambda_{0}$. Contrary to the standard opinion, the explanation of this remarkable fact is not found till now. The evidence to that of hundred publications in which various variants of the theory of effect under discussed up to day. Theoretical and experimental investigations of the Compton scattering mechanism, attempts to understand physical sense, proceed already almost century. This is unique phenomenon, since the effect has not obtained any significant practical application. In the theory of x-ray radiation scattering, this effect mention only with necessity to correct calculated atomic and structural scattering factors (along with other just incoherent effects - absorption, diffuse scattering, etc).

Such attention to Compton effect among experts is caused by two factors. The first: Compton effect has made the enormous influence on formation of quantum mechanics and quantum electrodynamics (Glauber, 2006). As it was already mentioned in introduction, it became a turning point in physics development, therefore any attempt to revise quantum physics inevitably should lead to attempts of revision of the accepted standard theory of Compton effect. The second factor. An explanation "softening" effect by collision of particles of point electron with photons broke the describing X-ray scattering theory of into "puzzles". Whole "image of an elephant" has broken up to images of its "foot", "trunk" and "body". Instead of common theory of scattering three various theories which are only formally agreed among themselves have come. Actually, in them are used various representations about entity of matter, various theories of atom structure, various mathematical apparatus. In the next paragraphs we will consider the theory based on de Broglie representation about the electron occupies all space and Schrödinger wave $\psi$-function as real fields. We will name this theory neoclassical (Crisp, 1990). This name reflects its essence more successfully than semiclassical theory, used various authors.

\section{Neoclassical theory of coherent X-ray scattering by bound electrons}

For an explanation of results of electromagnetic scattering on the bound electrons, Schrödinger, for the first time, interpreted the wave function $\psi$ found him as the function defining density of a charge in atom. According to his "electrodynamic" hypothesis, the charge density in atom in stationary condition is defined by the formula $\rho=e|\psi(r)|^{2}$. The elementary charges representing an electron in atom, oscillate under the influence of electromagnetic radiation. At such understanding of a charge, scattering on an electron in atom is reduced to a problem defining the function of scattering $f(H)$ for the big electron (7). Because all electrons in atom are in the central field and all charges density distributions are symmetric about centre, it is obvious, that scattering of each electron is coherent with others. The factor of atomic scattering $f_{\mathrm{a}}=\sum_{i=1}^{Z} f_{i}$, where $\mathrm{Z}$ - atomic number. Usual opinion: there is exist experimental prove that all electrons scatter coherently according to Schrödinger $|\psi(\mathrm{r})|^{2}$ representation for each electron. This opinion is widely used in X-ray structure analysis. At the same time, as already was point above, it has been shown, that electrons in atoms of easy elements scatter incoherently with each other, and their size much less than defined for an electron by function $|\psi(\mathrm{r})|^{2}$. 
Actually, as we can see from analysis of the Fig. 3, all electrons simultaneously participate both in Rayleigh, and in Compton scattering. It is possible to consider approximately, that factor of coherent scattering by electrons in atoms of light elements is $R_{\mathrm{ch}}=I_{c h} / I_{t h}$, where $I_{c h}$ and $I_{\text {th }}$ intensity of coherent and Thomson scatterings.

Evidently, the same electrons participate in forming both part of spectra. It is obvious, that charges in atom scatter coherently should remain motionless and occupy great volume of atom. So there are no alternatives of function interpretation $|\psi(r)|^{2}$ except real distribution of charge density.

It is necessary to assert, that electrons in atom remain motionless. Therefore standard model of atom, with electrons are point particles having an impulse corresponding to velocity $10^{-2} \mathrm{C}$ - $10^{-1} \mathcal{C}$ where $c$ - velocity of light, is incorrect from point of view of X-ray diffraction experiment. Otherwise, coherent, unmodified part of spectrum would not be observed at all for moving electrons. In the case of standard model, separate atoms even united in a crystal lattice, will give smoothed diffraction picture (like diffuse thermal scattering), as for it formation it is necessary synchronous moving electrons in atoms of a crystal lattice. But also in this case the atomic scattering factor will be differs from calculated from formula (6), because instead of addition scattering amplitude it is necessary to sum up intensity of it scattering from points, defined by function ${ }^{2}|\psi(r)|^{2}$. "Electrodynamic interpretation" gives some distribution of electronic density in atom, but does not explain loss more than $90 \%$ of a scattering matter in atoms of light elements (intensity of "incoherent" scattering much higher than "coherent" one). Above noticed, the condition (1) - basic condition both valued for classical electrodynamics and NCT. Hence, only a part of the electronic mass, located in an electron, provide Rayleigh scatterings. Designate this mass $m_{a}$. It is obvious, that it makes a small part of a lump of an electron. Other part, we name "dark matter" $m$ ', scatter "incoherent", forming Compton spectrum.

Quantum theory of scattering explain existence this "dark matter" in atom only that the part of electrons is pulled out from the position determined by function $|\psi|^{2}$ for very short time, an order $\mathrm{t} \approx 1 / \mathrm{v}_{0}$ (Cooper, 1985). Other electrons "do not notice" this loss and keep the previous position. The pulled out electron scatter radiation on the mechanism of incoherent scattering, which we will discuss in the next paragraph. We will notice only, that this electron "recollects" an impulse, which ostensibly was in atom. This is one more "ad hoc" hypothesis of quantum theory of x-ray scattering.

Let's quote Compton reasoning about the discrete electrons scattering concept which reflects the standard point of view on the mechanism coherent scatterings: "According to the wavemechanics theory, under the influence of the field of the incident electromagnetic wave the characteristic functions for higher energy states of an atom assume finite values and the radiation which it emits has the frequencies described by $h v_{0}=h v+W_{i}-W_{f}$ (where $W_{i}$ and $W_{f}$ are the initial and final energies). If the final state of the atom is identical with the initial state $i$, the frequency is unchanged, and coherent radiation is emitted. In calculating this part of the scattering, only the $\psi$ functions of the normal state 0 of the atom are therefore concerned. That is, the coherent scattering is identical with that from an atom having a

2The similar situation considered Bosanac in article where analyzed application consequences semiclassical theories for the analysis of Compton lines (Bosanac, 1998). 
continuous distribution of electric charge of density $\rho=-e \psi_{0} \psi_{0}^{* \prime \prime}$ (Compton, 1935). Such reasoning naively reduced to false statement, that the probability of a finding of a point electron in volume $\mathrm{dV}$ is equivalent to distribution of a scattering charge. From the point of view of the quantum theory of a structure of atom, neither orbital movement, nor any another movement of electrons in atom outwardly should not be shown, as the electron cannot lose energy, being in a stationary condition. From experiments on coherent scattering it is possible to draw a conclusion, that movement of electrons in atom actually is not exist and invisible. We will show now, how it is possible to "remove" electron movements in atom, having real, explicit physical sense "nonobservability" of them.

Schrödinger to find $\psi$-function placed the electron in a force field of atom, and electron get an impulse

$$
p=\sqrt{2 m(\varepsilon-V)}
$$

where $V$ - a force field potential.

Lets the stationary condition has some energy $E_{i}$. Electron-binding energy $E_{i}$ defines an impulse $p_{i}=\sqrt{2 m E_{i}}$ or de Broglie wavelength

$$
\Lambda_{B i}=\frac{h}{p_{i}}=\frac{h}{m \vartheta}
$$

We will consider, that "coherently" scattering "part" of an electron, mass $\mathrm{m}_{\mathrm{a}}$, is proportional to $R_{c h}$. It is natural to suppose, that this relation is equal to

$$
R_{c h}=\frac{m_{a}}{m}=\frac{p_{i}}{m c}=\frac{\Lambda_{e}}{\Lambda_{B i}}=\sqrt{\frac{2 E_{i}}{m c^{2}}}=\alpha_{i},
$$

For "incoherent" ("dark") mass $m^{\prime}$, forming a Compton spectrum part, it is possible to obtain, using the same logic, the result: the bound electron gets impulse $p_{i}=\sqrt{2 m E_{i}}$. Compton wavelength $\Lambda_{\mathrm{e}}$ and mass $m$ of a free electron changes on the values $\Lambda_{\mathrm{e}}^{\prime}$ and $\mathrm{m}^{\prime}$ in the manner:

$$
\Lambda_{e}^{\prime}=\frac{h}{m c+p_{i}}=\Lambda_{e}\left(1-\alpha_{i}\right), m^{\prime}=m\left(1+\alpha_{i}\right)
$$

Try to estimate factor of scattering by atoms of easy elements:

$$
\begin{aligned}
& \mathrm{R}=\frac{\mathrm{I}_{\mathrm{ch}}+\mathrm{I}_{\text {in }}}{\mathrm{I}_{\text {th }}}=\alpha^{2}\left|\mathrm{Z}-\sum_{\mathrm{i}=1}^{\mathrm{Z}} \frac{\alpha_{\mathrm{i}}}{\alpha} \mathrm{f}_{\mathrm{i}}\right|^{2}+\left(\mathrm{Z}-\alpha^{2} \sum_{\mathrm{i}=1}^{\mathrm{Z}}\left|1-\frac{\alpha_{\mathrm{i}}}{\alpha} \mathrm{f}_{\mathrm{i}}\right|^{2}\right), \\
& \alpha=\sum_{\mathrm{i}=1}^{\mathrm{Z}} \alpha_{\mathrm{i}}
\end{aligned}
$$

\footnotetext{
${ }^{3}$ The value $R$ was estimated by Compton and other authors (Compton, 1935) from the consideration of quantum derivation for "incoherent" part scattering spectra. Their results rather differ from ours. Significance and sense of this fact will be explain in the next paragraphs.
} 
This estimation is valid only for the scattering angles outside of Bragg conditions. In the case of Bragg diffraction on crystal structure only coherent radiation in $\varphi$ direction is observed.

Thus, coherent Rayleigh scattering of electromagnetic radiation is possible only by bound electrons. Bound energy of electrons in atoms of light elements is small in comparison with $m c^{2}$ and it is enough to localise near to atom only small part of electronic mass of each electron. Interpretation $|\psi(r)|^{2}$ as a real function lead to necessity to replace momentum representation $\vec{p}_{i}$ on de Broglie wavelength $\Lambda_{\mathrm{B} i}$ representation. Distribution $\chi(p)$ momentum distribution in atom, is a Fourier transformation of $\psi(r)$ function (Dirac, 1926), also necessary replace by de Broglie wavelength distribution:

$$
\chi(\vec{p})=\chi\left(\frac{h}{\Lambda_{B}}\right)=\left(\frac{h}{2 \pi}\right)^{\frac{3}{2}} \int \psi(r) \exp \left(-i 2 \pi \frac{r}{\Lambda_{B}}\right) d r
$$

This equation gives clear physical sense to why "momentum" of electrons being in atom is not visible at coherent scattering of x-ray radiation. Such understanding of "coherent" x-ray scattering give us possibility to regard "incoherent" scattering actually as partly coherent and changes a view at structure of a free electron. We will consider this questions in details.

\section{Wave-particle model of free electron in "electrodynamic interpretation" as real wave packet electrical charge density}

In the previous section we pointed to the contradiction between an explanation of coherent scattering in " electrodynamic interpretation " $|\psi(r)|^{2}$ function with concept of point electron. We made a choice in favour of Schrödinger $|\psi(r)|^{2}$ interpretation as the main concept, in spite of usually auxiliary considering, helping to understand physical sense of an event. Even Crisp, one of authors of the neoclassical theory, writes: "Schrödinger's interpretation of $\psi$ was found to be seriously flawed when used to explain the behavior of a free particle. The general spreading of a particle's wave packet made it too transient to be as stable as particles that are found in nature. Furthermore, the application of this interpretation of quantum mechanics to a scattering experiment suggest a splitting, or division, of particles which is not experimentally observed" (Crisp, 1990).

The true reason of seeming "splitting" of an electron in experiments on x-ray radiation scattering lays in misunderstanding of the free electron state description. Schrödinger equations can be received in nonrelativistic approach at $\nu_{\mathrm{B}}<<\nu_{\mathrm{e}},\left(\right.$ or $\left.\Lambda_{\mathrm{B}}>>\Lambda_{\mathrm{e}}\right)$, where

$v_{B}$ - any frequency of function $\psi(r, t)$. In this case it is possible to consider, that $\psi$ function is slow envelop function of high-frequency relativistic function $\exp \left(i 2 \pi v_{\mathrm{e}}\right)$.

It is known, that each electronic de Broglie wave satisfies Klein-Gordon equation

$$
\nabla^{2} \Phi-\frac{1}{c^{2}} \frac{\partial^{2} \Phi}{\partial t^{2}}=\left(\frac{m c}{h}\right)^{2} \Phi
$$


Plain wave decision of this equation is

$$
\begin{aligned}
& \Phi=\Phi_{0}(r, t)=\exp 2 \pi i\left(k_{0} x-v_{0} t\right), \\
& k_{0}=0, v_{0}=v_{e}
\end{aligned}
$$

Let's $\Phi(r, t)=\psi(r, t) \exp 2 \pi i v_{e}$, then at the conditions $v_{B}<<v_{e}$, we can neglect member $\ddot{\psi}$ and write down approximately:

$$
i h \dot{\psi}=-\frac{h^{2}}{2 m} \nabla^{2} \psi+\left(\frac{h^{2}}{m c^{2}} \ddot{\psi}\right) \approx \frac{h}{2 m} \nabla^{2} \psi
$$

Let's pay attention to that difficulty ("serious flaw") in Schrödinger interpretation connected with interpretation $\psi$ as a real wave of electronic density at excitation of an electron at rest by an electromagnetic wave. The frequency $v_{\mathrm{e}}$ in this case are not localised, but synchronous everywhere. In such interpretation of the wave process inherent to a rest electron, de Broglie wave of a moving electron automatically become waves of probability, which describe electron movement at a big distance $R$ from it $\left(R>>\Lambda_{e}\right)$ in a far zone, and do not give the information on its real position. At movement of an electron with a velocity $\vartheta$ in a direction $r$ in fixed coordinate system, the de Broglie wave is:

$$
\sin 2 \pi v_{e}\left(\frac{t-\frac{\vartheta}{c^{2}} r}{\left(1-\beta^{2}\right)^{\frac{1}{2}}}\right) \equiv \sin 2 \pi \frac{r}{\left(1-\beta^{2}\right)^{\frac{1}{2}}}\left(\frac{1}{\Lambda_{e}}-\frac{1}{\Lambda_{B}}\right)
$$

Phase velocity of process of field propagation $V$, de Broglie wavelength $\Lambda_{e}$ and $\beta$ are:

$$
V=\frac{c^{2}}{\vartheta}, \Lambda_{B}=\frac{h}{m \vartheta}, \beta=\frac{\vartheta}{c},
$$

By analogy with paragraph 3, replace time fluctuations with frequency $v_{\mathrm{e}}$ a of a rest electron, on the spatial fluctuations formed by real waves. For this purpose it is necessary to image, that two counter electronic waves - outgoing and ingoing, entering in a point $r=0$, and formed a standing wave of electronic density. If the length of each of this waves is equal to $2 \Lambda_{e}$, the electronic density for a free electron at rest is described by set of plane waves. In any direction $\vec{r}$ is a wave of charge density:

$$
\begin{aligned}
& \rho(R) d \tau=e|\psi(R)|^{2} R^{2} d R d \Omega, \\
& R=\frac{r}{\Lambda_{e}}, d \tau=\frac{d V}{\Lambda_{e}^{3}}
\end{aligned}
$$

where

$$
|\psi(R)|^{2}=\frac{\sin ^{2}\left(\frac{\pi r}{\Lambda_{e}}\right)}{\left(\frac{\pi r}{\Lambda_{e}}\right)^{2}}=\frac{\sin ^{2} \pi R}{\pi^{2} R^{2}}=\frac{(1-\cos 2 \pi R)}{2 \pi^{2} R^{2}}
$$


It means, that in any direction $\vec{r}$ at $\mathrm{r}>>\Lambda_{\mathrm{e}}$ the wave de Broglie a rest electron with spatial frequency $\vec{k}=\Lambda_{e}^{-1}$ are exist. At electron movement with a velocity $\vartheta$ wave process is described by both representation in the formula (18), but physical sense has only real wave:

$$
\sin 2 \pi \frac{r}{\sqrt{1-\beta^{2}}}\left(\frac{1}{\Lambda_{e}}-\frac{1}{\Lambda_{B}}\right)
$$

This movement are described by standing wave of electronic density movement and by "in" and "out" waves moving with relative velocity $\vartheta=\beta c$ each of them receive usual Doppler shift (Wolf, 1998).Phase velocity in a movement direction in a far zone corresponds to usual de Broglie waves as in (18), but now concrete sense is given to these waves. Electron movement in this consideration should be described not by single plane wave spreading in $x$ direction, but by all plane waves, in a point $x=\vartheta_{x} \mathrm{t}, \mathrm{y}=0, \mathrm{z}=0$. In such representation both moving and rest electrons as de Broglie waves, Klein-Gordon equation gets sense of the equation of a real plane waves. It is obvious, that all wave structure of an electron scatter an electromagnetic field under the complex law, and leads to a two-part spectrum. "Coherent" part is formed as a result of deformation phase periodic structure of an electron. This elastic deformation leads to appearance of small homogenous electron charge distribution described by Schrödinger equation. In the next paragraph, we try to consider Compton effect as effect of unusual coherent scattering. At the same time, wave structure of atomic electron is deformed according (15).

\section{Compton effect as effect of coherent scattering}

Fig 4 represents the scheme, suggested by Compton, explaining effect as incoherent scattering. The primary photon with energy $\mathrm{h} \nu_{0}$ is absorbed by a point electron. The new particle, possessing total energy and an impulse of a photon and an electron is formed. For a short time interval (it is sometimes supposed about time of an order $1 / \nu_{0}$ ) this quasiparticle breaks up on two - a new photon with energy $\mathrm{h} v^{\prime}<\mathrm{h} v_{0}$ and an electron with some kinetic energy. Writing down the energy and impulse conservation laws it is easy to connect energy and impulses of the new particles. In case of scattering by free electron formula (12) describes relationship between frequencies and wavelengths of the primary and secondary photons. Formula (12) allow to define only centre of line, explanation of wide width of scattered lines, not envisioned in Compton scheme, offered Jauncey (Jauncey, 1925). In 1933 DuMond has published the theory is known as impulse approximation in which the width of the line is compared with those impulses $\vec{p}$, which the electron has in atom in the bound state, as he guess (DuMond, 1933).

In his theory, Doppler shift provide line spreading. In common, most physicists grounded in classical electrodynamics were a grudging acceptance for Compton explanation of "incoherent" scattering effect. However, the twentieth of 20 century was turbulent period in the evolution of physics. Compton effect was "turning point of physics" (Stuewer, 1975) and pull the trigger of a general acceptance of quantum ideas. It is possible to agree with this estimation of Compton effect, its treatments by Compton, but possible to ask a question: whether on a correct way physics development, since this point has gone. For this purpose 
once again we will analyse Compton, DuMond and other founders of the theory "incoherent" scattering effect arguments from the neoclassical theory point of view. It is necessary "to combine all puzzles" experimental results X-ray radiation scattering from Barkla to Compton in a whole picture.

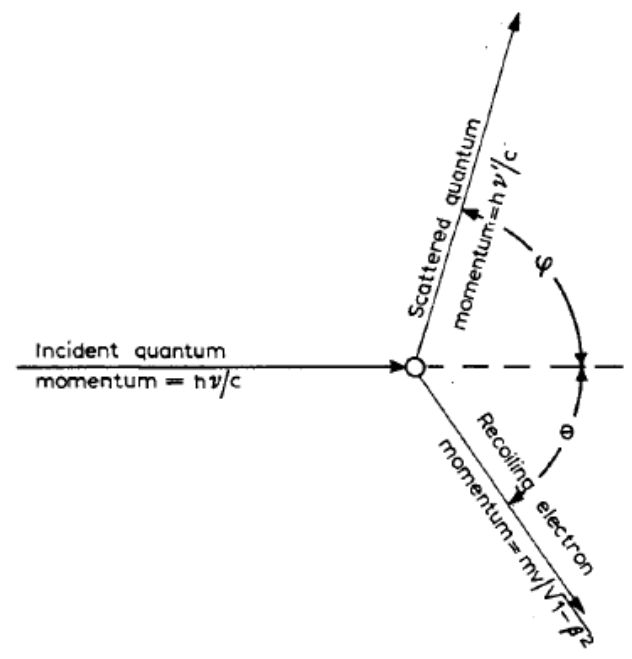

Fig. 4. An X-ray photon is deflected through an angle $\varphi$ by an electron, which in turn recoils at an angle $\theta$, taking a part of the energy of the photon. (Compton, 1923, 1927)

\subsection{Scattering by a free electron (early models of Compton effect)}

Behind seeming simplicity and grace of the modern Compton effect theory, stand some "ad hoc" hypotheses, which has put difficulties, contradictions and "obscurity" the quantum physics, mentioned in introduction. Was used the hypothesis: reduction or a collapse of wave function (in this case, an x-ray electromagnetic wave), i.e. instant transformation wave in a particle. It contradicted to previous physical experience: this particle-photon get not only the energy received by it at a "birth" but impulse $\vec{p}$, directed from a source to a concrete electron. Clearly, already at this analysis stage theory need to add two new, very strong new hypotheses to the earlier quantum hypotheses: Planck (atom radiates quanta of energy $E=h v_{0}$ ) and Einstein (absorption of energy by matter occurs also discretely to energy $\mathrm{h} v_{0}$, equal some resonant energy).

First from new hypotheses: absorption of energy is accompanied by absorption of impulse quantum.

Second hypothesis: electron radiate just new photon, but not radiation, and wave function of the photon is only the function describing probability of its occurrence in concrete point of space - a collapse at a "birth".

Compton theory is a remarkable example of replacement in quantum electrodynamics of the physical sense in interaction of an electromagnetic field with charged particles, by the 
mathematical fictions, and hypotheses. Really, in Compton theory electromagnetic interaction between photon an electron is considered only at determination of probability photon scattering by an electron which is proportional to the scattering cross section $\sigma_{0}$, calculated in the classical theory. Wonderfully, in Compton theory there is no consideration of the processes occurring in a time interval between the beginning of interaction of radiation with an electron and its finishing. In introduction it was noticed, ignoring of transient process typical for all quantum electrodynamics and is very important feature influencing on interpretation of particles interaction process. In the case of Compton effect, removed the process occurring at interaction of radiation with electrons, we removed such physical processes, as: electron acceleration, appearance of Lorentz force radiating braking. Their account in classical electrodynamics leads to a conclusion that the electron should remain motionless at scattering of a wave.

Schrödinger criticized the Compton scheme of formation radiation spectrum with the shifted wavelength and pointed out the discrepancy with the electromagnetic theory (Schrödinger, 1927). He offered model in which the electromagnetic wave scatters on moving de Broglie real wave. In this model, at correctly chosen velocity of an electron, frequency of a primary wave gets the shift corresponding to the formula (12). Later Dodd investigated this problem as the problem of plane electromagnetic wave scattering on a moving electron (Dodd, 1983).

According to the Dodd model of Compton scattering, the electron moves with a velocity

$$
\vartheta=\vartheta_{0}\left(\frac{c}{c+\vartheta_{0}}\right), \text { where } \vartheta_{0}=\frac{h v_{0}}{m_{e} c}
$$

The plane electromagnetic wave with frequency $v_{0}$ propagate in a direction parallel to movement of electron. Lorentz transformations at scattering on such electron lead to frequencies and wavelengths value equal to (12). Neither Schrödinger, nor Dodd, did discuss reason of electron movement. They only postulated necessity of electron movement to receiving angular distribution of frequency of scattering wave.

As we demonstrate above, scattering on a free electron should not lead to absorption neither energy, nor an impulse. The free electron has no possibility to take impulse from electromagnetic radiation, it should remain motionless, making under the influence of radiation only periodic oscillations. All experience of X-ray structural analysis shows, electrons in atoms keep the position at scattering. The photoeffect, other effects of incoherent scattering at which there is a displacement of electrons from stationary position, are connected with interatomic absorption. These secondary effects are absent at scattering on free electrons and are small at scattering of hard radiation on atoms of easy elements. Compton effect is effect of scattering on a free electron and dominates at scattering on atoms of easy elements, but nevertheless looks like effect being in close connection with point electron movement. From that consideration we have to confirm: Compton effect have to be regarded evidently as a experimental argument in favor of conclusion about real wave particle model of free electron, taking some volume in space. Linear sizes and details of it structure are comparable to $\mathrm{X}$-ray radiation wavelengths. 


\subsection{Neoclassical theory of scattering by free electrons}

Let's note once again: the base for Thomson's classical theory of X-ray scattering on a free electron is the assumption about physical pointness an electron as particle with the size $r_{e}$.

The theory admits also possibility of existence of the "big" electron with the sizes of order $\Lambda_{\mathrm{e}}$, with relation of a charge to mass in each elementary volume equal to e/m (1). Quantum electrodynamics starts with mathematical model of a point electron, though the condition Schrödinger equation is derived limits the size of "point" equal to $\Lambda_{\mathrm{e}}$.

This restriction is known as a Heisenberg uncertainty principle, and it reflects not character of basic impossibility to define coordinate of an electron (more precisely, than this size), but fundamental statement, that the charge and mass of an electron which occupies all space (as represented still de Broglie) can mainly take place in the particle with diameter $\Lambda_{\mathrm{e}}$.

In the previous paragraph we represent the function $\cos 2 \pi R$ as function for describing of electronic density distribution in a free electron in any direction $\vec{r}$. Let electromagnetic wave propagates along periodic distribution of electronic density in direction $\vec{n}_{0}$. In this case, oscillation along electronic structure moves with a velocity $c$ followed electromagnetic wave in direction $\vec{n}_{0}$. Such scheme is completely equivalent Dodd scheme, but electron movement replaced by propagation electronic waves along electronic structure. It means that along standing wave electron density moves toward each other with a velocity of light two waves. Phase shift between them is equal to $4 \pi \Lambda_{\mathrm{e}} / \lambda_{0}$.

In other words, along an electron two transverse waves (excitations) propagate with a velocity of light, that causes two dipole electromagnetic waves, shift of phases between them in directions $\vec{n}_{\varphi}$ leads to angular change of wavelength (the same shift received in theories Compton, Dodd and Schrödinger):

$$
\Delta \lambda=c \Lambda_{e}\left(\frac{1}{c}-\frac{\cos \varphi}{c}\right)
$$

Note, at derivation an equation (21) we explain both Compton shift and also physical sense of mechanism of formation Lorentz radiating force (11). Along an electron with periodically distributed electronic density the phase wave do not transfer an impulse - the electron remains motionless. Fig.5a presents model of an electron (19) - a standing wave of electronic density with spherical symmetric periodic distribution. Let's now the plane electromagnetic wave $\vec{n}_{0}$ propagate under any angle $\varphi$ to the plane electronic wave in direction $\vec{n}_{\varphi}$ (Fig. 5b). It causes in direction $\pm n_{\varphi}$ two opposite transverse electronic waves and two sets of dipole electromagnetic waves. Phase shift between in-out dipole waves formula (21) describes.

Thus, the electronic structure with distribution of electronic density (19) radiates unusual "composite" dipole wave. The wavelength of this radiation depends on an angle $\varphi$. The width of spectrum $\delta \lambda=\Lambda_{e}(1-\cos \varphi)$, that is coherency, is defined by a spectrum of spatial frequencies of function sinc ${ }^{2} \pi r$ at any direction $\varphi$. 

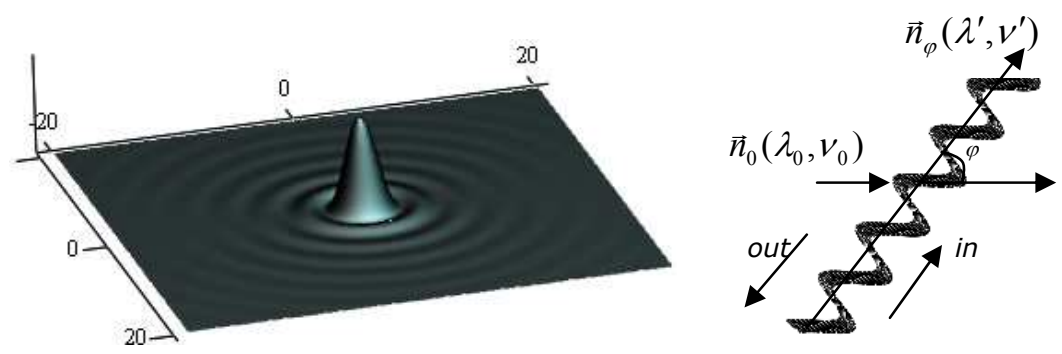

Fig. 5. a) Schematic radial distribution of charge density in electron $\rho(r)$ (19), (20). De Broglie wavelength in any direction $\vec{n}_{\varphi}$ is equal to $\Lambda_{e} ; \mathrm{b}$ ) wave scattering in one of direction $\vec{n}_{\varphi}$. Inout electronic waves run along $\vec{n}_{\varphi}$ direction. Phase velocity of propagation of electron excitation is equal to $\mathrm{c} / \cos \varphi$.

\subsection{Neoclassical theory of scattering by bound electrons in light atoms}

Theoretical distribution of intensity Compton line for free electron at the angle $\varphi=90^{\circ}$ and angular interval $0^{\circ}<\varphi<180^{\circ}$ is presented on Fig.6a,b.
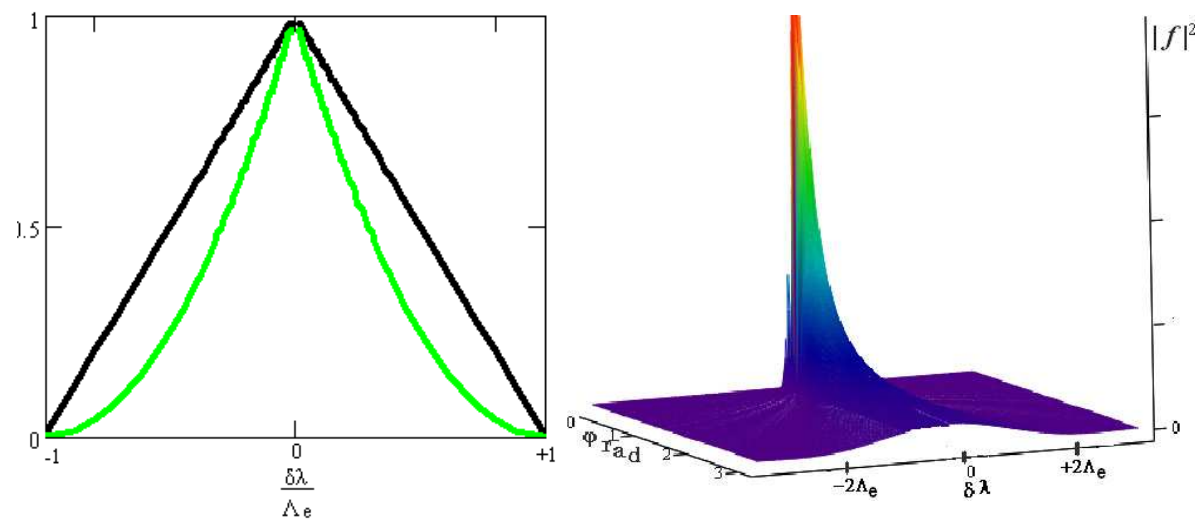

Fig. 6. a) Black line: Fourier spectra for $|\psi|^{2}$; color line: intensity distribution $|f(\varphi, \delta \lambda)|^{2}$ for "incoherent" scattering by free electron at $\varphi=90^{\circ}$; b) Theoretical dependence $|f(\varphi, \delta \lambda)|^{2}$ for "incoherent" line in angular range $0^{\circ}<\varphi<180^{\circ}$

On Fig.6a black line is Fourier spectra of $|\psi(r)|^{2}$ function for free electron (20):

$$
F\left[|\psi(r)|^{2}\right]=f\left(\frac{\delta \lambda}{\Delta \lambda^{\prime}(\varphi)}\right)
$$

at $\varphi=90^{\circ}, \Delta \mathcal{U}^{\prime}(\varphi)=\Lambda_{\mathrm{e}}$; color line is intensity of this spectra $|f(\varphi)|^{2}$.

Integral intensity of scattering at $\lambda_{0}>>\Lambda_{\mathrm{e}}$ equal to classical one (scattering factor $R=1$ ) both for point-like and wave-particle (20) electron, and independent of angle $\varphi$. Fig.6b presents calculated distribution $|f(\varphi, \delta \lambda)|^{2}$. 
At calculations we assumed, that $\lambda_{0}>>\Lambda_{e}$ (we compare calculation to experiments results with $\lambda_{0}=0.88 \AA$ ). A case $\lambda \geq \Lambda_{\mathrm{e}}$ we consider later. Let's remind, since work DuMond, width of Compton lines someone try to explain as electron (has been beaten out from atom) save velocity that it had in the bound state, i.e. impulse $\vec{p}$ from distribution $\chi(\vec{p})$ (15). New photon, forming by scattering on such free electron has Doppler shift of frequency. Since $p_{\max }=\sqrt{2 E_{i} m}$ the width of a line should be defined by size $\mathrm{a}_{i} \lambda_{0}$. This width in case of scattering on carbon comparable to the width of the Compton line for free electron. Therefore, DuMond, theory seemed plausible, and experimental checks of "impulse approximation" continue in the same spirit till now. Actually, the change of width of Compton line from bound electron comparable with a width of line from free electron. The difference have to be very small and is order to $\delta \lambda=\Lambda_{\mathrm{e}}-\Lambda_{\mathrm{e}}^{\prime} \approx \mathrm{a}_{i} \Lambda_{\mathrm{e}}$ (See (13)). This value is much less than in the quantum theory accepted today. In the case of scattering on carbon atoms, even for K-electron $\left(\mathrm{E}_{i} \approx 280 \mathrm{eV}\right), \alpha_{i} \approx 3 \cdot 10^{-2}$. It means, that the width and the spectrum form of Compton line on carbon is defined by scattering on free electron within several percent accuracy. Fig. 7 represents the spectrum of scattering on a diamond monocrystal in angular interval $10^{\circ}-160^{\circ}$. We see, line form qualitatively coincides with the calculated (Fig 6b).

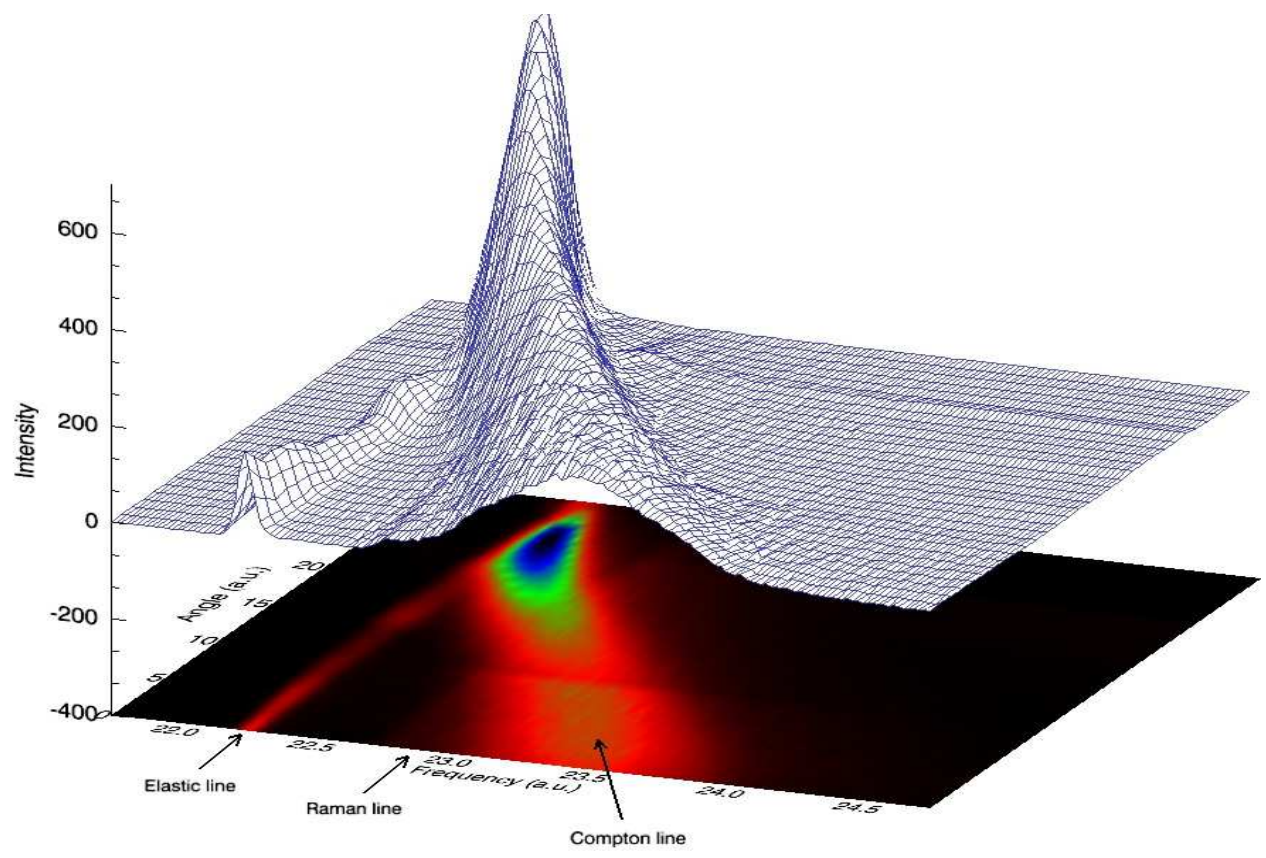

Fig. 7. Experimental spectra at $\lambda_{0}=0.88 \AA$ on a diamond single crystall, at various angles $\varphi$ from $10^{\circ}$ to $160^{\circ}$. Among "coherent" (elastic) and "incoherent" (Compton) lines, is weak Raman (J-line; about reason for term $h v_{\text {J }}$ for Raman-line more detailed in paragraph 6). 
Fig. 8a presents dependences: theoretical (color line) and experimental (points) full width at half intensity of a line $|f(\varphi, \delta \lambda)|^{2}$. Fig.8b presents theoretical (color line) and experimental results (points) intensity in a Compton lines maximum $|f(\varphi, 0)|^{2}$.
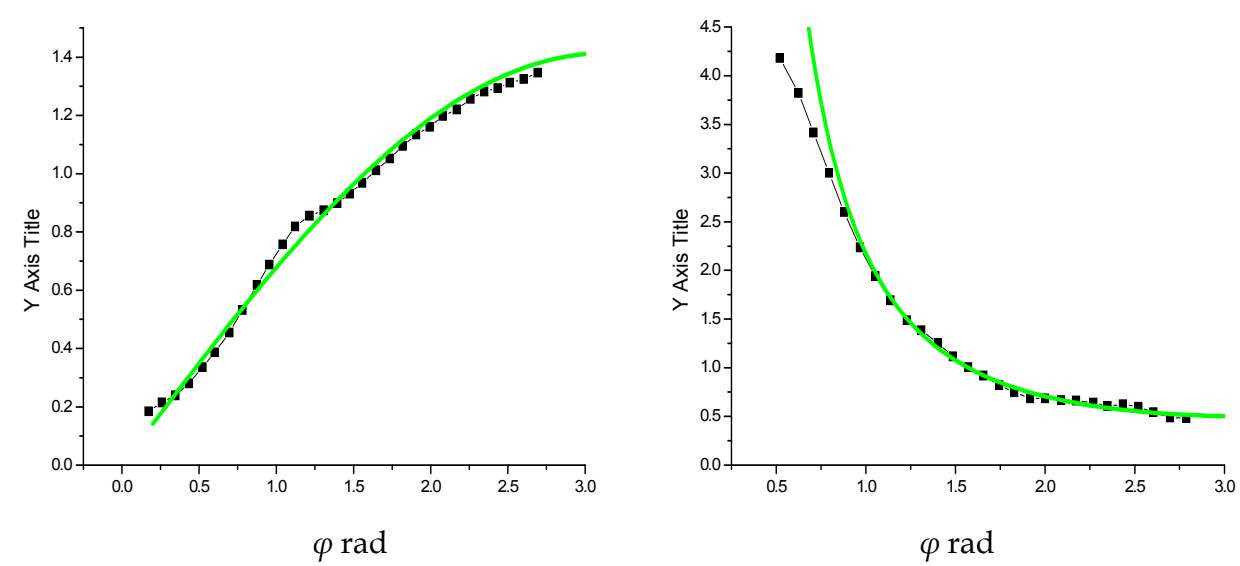

Fig. 8. a) Full width at half intensity of a line $|f(\varphi, \delta \lambda)|^{2}$ of "incoherent" line (for Fig.7) (in arbitrary unit). Points - experimental date; color line theoretical $\delta \lambda \sim(1-\cos \varphi)^{1 / 2}$; b) Intensity dependence in maximum (center) of "incoherent" line (for Fig.7) . Points -experimental date; color line - theoretical intensity dependence $I_{\mathrm{m}} \sim(1-\cos \varphi)^{-1}$. Y-axis in arbitrary units.

It is clear, these dependences well coincide. However, similar measurements on monocrystals $\mathrm{Si}$ show much less coincidence from calculated for a free electron (for kelectrons in $\mathrm{Si}, \alpha_{i} \approx 1.2 \cdot 10^{-1}$ ).

Theory of impulse approximation evaluate some displacement (Ross \& Kirkpatrick, 1934) for centre of Compton line on value

$$
\Delta \lambda=-\frac{\lambda_{0}^{2}}{\mathrm{hc}} \frac{1-\mathrm{n}}{\mathrm{n}} \mathrm{E}_{\mathrm{i}} ; \mathrm{n} \approx \frac{1}{2}
$$

This estimation show, that impulse approximation theory predicts for displacement of Compton shift

$$
-\frac{\Delta \lambda}{\lambda_{0}}=\frac{\Delta v}{v_{0}}=\frac{v_{i}}{v_{0}}
$$

This value derived under assumption of short collision of photon with electron. From another point of view, according to energy conservation law this line displacement have to be equal to usual equation for position of Raman X-ray line (J-line) (Raman, 1928; Pimpale \& Mande, 1984) and do not observed as Compton line shift. In this case frequency change:

$$
h v_{J}=h v_{0}-E_{i}=h\left(v_{0}-v_{i}\right) ; \Delta v=v_{i}\left(v_{0}-v_{J}\right)
$$


At the analysis of Compton scatterings consider, that frequency change $\Delta v(\varphi)$ should be much less $v_{i}$. This condition is necessary to explain experimental fact, that the shift of a line given by the formula (22), not observed. Experimentally we see J-line (Raman line) inside the Compton scattering spectra (see Fig.7). It means, at all angles, $\Delta v(\varphi)$ comparable to $v_{i}$. Frequency $v_{i}$ for $K$-electron in carbon corresponds to energy excitation $280 \mathrm{eV}$. Frequency change $\Delta v$ at $\varphi=90^{\circ}$, corresponds to energy change approximately equal to $380 \mathrm{eV}$.

According to table data (Alexandropoulos et al., 2004) under the experimental condition incoherent scattering factor is more than 5 in wide angle $\varphi$ interval. It means, that all 6 electrons in carbon should give the contribution to "incoherent" scattering. Nevertheless, the line centre is not displaced, that once again to get support to discussed model of scattering.

The mechanism of formation of a Compton spectrum, considered above, has been offered for the first time by V.Aristov in 2008 (Aristov, 2009b) and discussed in a conferences (Aristov, 2010, 2011). At first sight, suggested mechanism is unusual. However, in optics of visible light, radio optics, it is possible to find examples of formation of the angular wavelength radiation dependence. The nearest analogy to Compton effect - Purcell-Smith effect (Smith \& Purcell, 1953), and especially inversed Purcell-Smith effect, was suggested for electron accelerators (Kim, 1993). Purcell-Smith effect is a radiation caused from electron movement with a velocity $\vartheta$ along a dielectric lattice with the period $b$. Angular dependence radiated electromagnetic wavelength is:

$$
\lambda=\frac{b}{\beta}(1-\beta \cos \varphi)
$$

The wavelengths difference between direction $\varphi=0$, and all others is equal to:

$$
\lambda(\varphi)-\lambda_{0}=\Delta \lambda=b(1-\cos \varphi)
$$

\subsection{Compton scattering cross section}

One of the achievement of the quantum theory of $x$-ray radiation scattering is the theory explaining angular dependence of scattering factors from wavelength, based on works Klein and Nishina, Tamm (Klein \& Nishina, 1929; Tamm, 1930).

This dependence was discussed already in paragraph 2 (Fig 2). There is nothing surprising that results of the quantum theory correspond to that have been received by Compton in 1919 in frame of classical scattering theory. The quantum theory operates in space of impulses. In the kinematical theory of scattering (in the first Born approximation) the interference is described in space of reciprocal wave vectors also.

In the neoclassical theory scattering in each directions $\varphi$ is defined by independent oscillation by elementary charges. Each of them scatters as in classical electromagnetic theory. As was mentioned above, integral intensity at any angle $\varphi$ is constant at $\Lambda_{e}<<\lambda_{0}$, as well as in case of a point charge. In approximation of $\Delta v(\varphi)<<v_{i}$ it is possible to conclude that classical theory is true, an electron is point, and the scattering factor is proportional $r_{0}^{2}$. Moreover, "core" of an electron in the interval between the two first zero of function $\operatorname{sinc}^{2} \pi R$ 
forms an angular spectrum of quasi-monochromatic radiation in any direction. As a result of an interference this spectrum leads to angular dependence of integral intensity, as though in classical theory for "big" electron. Radius of "big" electron have an order of value $\Lambda_{\mathrm{e}}$. Possible to calculate a scattering spectrum using method, that Compton suggested in 1919, using various models of a big electron. Note, at large angles $\varphi$ "coherent" Rayleigh scattering it is possible to neglect and consider that all electrons in atom scatter as free, because $|\Psi(r)|^{2}$ area distribution much larger than $\Lambda_{\mathrm{e}}$.

\section{About true X-ray incoherent scattering}

The special section of physics is devoted to this topic and its consideration is not task of this chapter. However, we suspect the Compton "recoil" low-energy electrons do not connected with Compton effect, but with real absorption, i.e. with "true" incoherent scattering. In this chapter, under true incoherent scattering we consider X-ray Raman effect.

According to Einstein's hypothesis, the photoeffect reason is absorption of energy of light quantum $h v$, then electron overcome energy barrier that equal to binding energy and gets kinetic energy

$$
E_{k}=\frac{m \vartheta^{2}}{2}=h v_{0}-E_{i}
$$

In this equation there are no suggestions about concrete mechanisms of absorption and radiation. In this sense it nothing differs from the equations of conservation of energy and an impulse, written by Compton. In connection with such extremely superficial similarity "Compton" and "Einstein" effects are considered together not only in textbooks, but also in serious researches. Such identification of effects is incorrect. Photoelectric resonance absorption is responsible for photoelectrons. From this point of view, the photoeffect - is effect of classical electrodynamics (Lamb \& Scully, 1959). However, till now there is a debatable question how accumulation of energy quantum is carried out (Aristov, 2009a).

Compton theory is a theory for an explanation of effects of radiation scattering by free electron. However, any variants, except direct collision between point particles to transfer energy from one to another do not considered (even at scattering on the bound electrons). Instead of primitive billiard-like model, there is more complex and beautiful physical effect - scattering by infinite size electron with spherically symmetric distribution charge density. In this case, resonant absorption of radiation by bound electron is a more complicated phenomena, that is considered in classical and quantum electrodynamics. Excitation of electron gas produced due to photoelectric absorption lead to various secondary processes, including photoemission "Einstein-electrons" and "Comptonelectrons". Cross section of scattering and absorption by bound electron is determined by radiation braking factor

$$
\gamma=\frac{2 e^{2}}{3 m c^{3}} v_{i}^{2}, v_{i}=\frac{E_{i}}{h}
$$

In this case, instead of (3) movement equation of an electron: 


$$
\mathrm{V}(\mathrm{t})=-\frac{\mathrm{e}}{\mathrm{mc}} \overrightarrow{\mathrm{A}}(\mathrm{t}) \frac{1}{\left(v_{0}^{2}-v_{\mathrm{i}}^{2}\right)-\mathrm{i} v_{0} \gamma}
$$

The imaginary part of the equation (26) determines the photoabsorption, real part scattering cross section. Both scattering and absorption depends on frequencies $v_{0}$ and $v_{i}$ and from their difference $\left(v_{0}-v_{i}\right)$, that is equal of frequency of Raman scattering. Mechanism formation of this radiation only formally reminds optical Raman effect. We named this line as J-line. In our meaning, this line of radiation was observed Barkla and is named him a Jline (Barkla, 1917) by analogy to lines $\mathrm{K}$ and $\mathrm{M}$ characteristic radiation of atoms. It is remarkable, that Barkla and his opponents, including Compton, connected J-phenomenon with Compton scattering (Compton, 1924; Alexander, 1930).

$\mathrm{J}$ radiation arises because of energy absorption at frequency $v_{0}-v_{i}=v_{J}(22)$, and not because of exchange of energies between a photon $h v_{0}$ and quantum of orbital movement of an electron (such movement does not exist). The equation (26) has been written at assumption that electron is point-like. In our case, it is necessary to replace $v_{0}$ on $v^{\prime}(\varphi)$ (see (12)). It means, that absorption occurs at frequencies $v^{\prime}(\varphi)-v_{i}=\left[\left(v_{0}-\Delta v^{\prime}(\varphi)\right)-v_{i}\right]=v_{J}^{\prime}$.

In this case, for J-line, conservation energy law equation, combining (22) and (25), is:

$$
\mathrm{h} v_{\mathrm{J}}{ }^{\prime}=\mathrm{h} v_{0}-\mathrm{E}_{\mathrm{i}}+\frac{\mathrm{m} \vartheta^{2}}{2}
$$

Here $\vartheta$ - velocity of low energy "Compton" electrons, their appearance caused by incoherent J'-line. Finally, it is possible to write down the equations for a J-line and lowenergy electrons born with it:

$$
\begin{gathered}
v_{0}\left(1-\frac{2 \alpha}{1-2 \alpha}\right)<\left(v_{\mathrm{J}}+v_{\mathrm{i}}\right) \leq v_{0}, \quad \alpha=\frac{\Lambda_{\mathrm{e}}}{\lambda_{0}} \\
0 \leq \frac{m \vartheta^{2}}{2} \leq m c^{2}\left(\frac{2 \alpha^{2}}{1-2 \alpha}\right) \\
m \vec{\vartheta}=-\alpha m c \frac{\vec{H}}{\left|k_{0}\right|}
\end{gathered}
$$

values $\vec{H}, \vec{k}_{0}$ from (6). The equations (28) formally remind Compton equations. But physical sense considerably differ from them. The form of a J-line does not depend on an angle $\varphi$. It has the maximum energy at frequency $v_{J}=v_{0}-v_{i}$.

The J-line is wide, and its width $\Delta \lambda$ remains in good approach by a constant, and approximately equal to $2 \Lambda_{\mathrm{e}}$. With a J-line corresponding to K-electrons the lines corresponding to L, M-electrons should be observed.

The spectrum of low-energy electrons according to the formula (28) is qualitatively close predicted by Compton. Let's pay attention, that in formulae (25) - (28) constant $h$ is used only in the Planck sense. The bound electrons can radiate and absorb an electromagnetic wave according to classical electrodynamics laws. 


\section{Conclusion}

In the chapter, we formed and proved general conceptions of the neoclassical theory of interaction of electromagnetic radiation with matter on the free and weak bound electrons in atom. Neoclassical theory unifies all experimental scattering fragments in whole picture by means model infinite size electron. This model - standing spherical wave electron density with wavelength $\Lambda_{\mathrm{e}}$.

For such understanding all electromagnetic radiation scattered by an electrons, is coherent, that allows to suggest new methods for X-ray analysis and electronic spectroscopy. In thin films, can be realised methods of research similar Bragg diffraction, when incident radiation with $\lambda_{0}$ wavelength is reflects by the crystal under an angle $\varphi$ with $\lambda_{0}+\Delta \lambda(\varphi)$ wavelength (Aristov \& Shulakov 2010). It is interesting to measure Compton spectra (not only intensity) in schemes of standing waves. It is necessary to return, certainly, to Compton idea: investigation of the form and internal structure of an electron using hard radiation scattering diagrams (Compton, 1919). Possible to measure of a phase of the atomic scattering factor for integrated intensity of Compton spectra, as can see from (14).

\section{Acknowledgment}

This work was supported by RFBR, grant № 09-02-12090. The author also thank S.N. Yakunin for experimental results, and A.A. Despotuli for assistance.

\section{References}

Alexander, N. (1930). The J-phenomenon in X-rays. Proceedings of the Physical Society, Vol.42, No.2, (December 1929), pp.82-96

Alexandropoulos, N., Cooper, M., Suortti, P. \& Willis, B. (2004). Correction of systematic errors, In: International Tables for Crystallography. Vol. C., E. Prince, (Ed.), pp.653-661, Kluwer Academic Publishers, ISBN 1-4020-19009, Dordrecht, Netherlands

Akhiezer A.I. \& Polovin R.V. (1973). Why it is impossible to introduce hidden parameters into quantum mechanics, Uspekhi Fizicheskikh Nauk, Vol.15, (April 1973), pp. 500512, ISSN 0042-1294

Aristov, V. (2009a). The photoelectric effect in the semiclassical theory. Doklady Physics, Vol.54, No.4 (July 2008), pp. 171 -173, ISSN 1028-3358

Aristov, V. (2009b). Scattering of an electromagnetic wave on a free electron in the semiclassical approximation. Doklady Physics, Vol.54, No.4 (July 2008), pp. 187 -189, ISSN 1028-3358

Aristov, V. (2010) Fundamental problem of hard X-ray scattering (in russian), In: "X-ray optics". Working Conference. Chernogolovka-2010, pp. 208-211, IMT RAS, Available from http:/ / purple.iptm.ru/xray/xray2010/files/CHGXRAY2010_BOOK.pdf

Aristov, V. \& Shulakov, E. (2010) Bragg scattering due to changing of wavelength (in russian), In: "X-ray optics". Working Conference. Chernogolovka-2010, pp. 57-59, IMT RAS, Available from http://purple.iptm.ru/xray/xray2010/files/CHGXRAY2010_BOOK.pdf

Aristov, V. (2011). New Compton effect solution as the way to general x-ray scattering theory, Asia Pacific Academy of Materials-APAM. Abstract book. Taiwan, August, 2011 
Barkla, C.(1904).LXI. Energy of secondary Röntgen radiation. Philosophical Magazine Series 6, Vol.7, No.41, (May 1904), pp.543-560

Barkla, C. (1911). LXXVI. Note on the energy of scattered X-radiation. Philosophical Magazine Series 6, Vol.21, No.125, (May 1911), pp. 648-652

Barkla, C. (1918). Bakerian Lecture: On X-Rays and the Theory of Radiation. Philosophical Transactions of the Royal Society, A, Vol.217, No.549, (January 1918), pp. 315-360

Bell, J. (2004). Speakable And Unspeakable In Quantum Mechanics (second edition), Cambridge University Press, ISBN 052181862 1, United Kingdom

Bergman, D. (2004). Observation of the Properties of Physical Entities. Shape and Size of Electron \& Neutron, In: Common Sense Science, May 2004, Available from http:/ CommonSenseScience.org

Bohm, D. (1952) A Suggested Interpretation of the Quantum Theory in Terms of "Hidden" Variables. I. Physical Review, Vol.85, No.2, (January 1952), pp. 166-179

Bosanac, S. (1998). Semiclassical theory of Compton and photoelectric effects. The European Physical Journal D, Vol.1, (January 1998), pp. 317-327

Compton, A. (1919a). The Size and Shape of the Electron I. Physical Review, Vol.14, No.1, (March, 1919), pp. 20-43 Compton, A. (1919b). The Size and Shape of the Electron II. Physical Review, Vol.14, No.3, (May, 1919), pp. 247-259

Compton, A. (1922). Secondary Radiations Produced by X-rays, and Some of Their Applications to Physical Problems. Bulletin of the National Research Council, Vol.20, No.19, (October 1922), pp. 1-56

Compton, A. (1923) The Spectrum of Scattered X-Rays. Physical Review, Vol.22, No.5, (November 1923), pp. 409-413

Compton, A. (1924). Scattering of X-ray Quanta and J-phenomena. Nature, Vol.113, No.2831, (February 1924), pp.160-161

Compton, A. (1935). Incoherent Scattering and the Concept of Discrite Electrons, Physical Review, Vol.47, (March 1935), pp. 367-370

Compton, A. (1927). X-rays as a branch of optics, Nobel Lectures.

Cooper, M. (1985). Compton scattering and electron momentum determination. Reports on Progress in Physics, Vol.48, No.4, (April 1985), pp. 415-481

Crisp, M. \& Jaynes E. (1969). Radiative Effects in Semiclassical Theory. Physical Review, Vol.179, No.5, (March 1969), pp.1253-1261

Crisp, M. (1990) Self-fields in semiclassical radiation theory. Physical Review A, Vol.42, No.7, (October 1990), pp.3703-3717

Debye, P. (1909). Der Lichtdruck auf Kugeln von beliebigem Material. Annalen der Physik, Vol.30, No.1, pp 57-136

Debye, P. (1923) Zerstreuung von Rontgenstrahlen und Quantentheorie. Zeitschrift für Physik, Vol.24, (March 1923), pp. 161-166

Dirac, P. (1926) Relativity Quantum Mechanics with an Application to Compton Scattering. Royal Society's Proceedings A, Vol.111, (June 1926), pp. 405-423

Dirac, P. (1938). Classical Theory of Radiating Electrons. Royal Society's Proceedings A, Vol.167, (August 1938), pp. 148-169

Dirac, P. (1965). Quantum Electrodynamics without Dead Wood. Physical Review, Vol.139, No.3B, (August 1965), pp. B684-B690

Dodd, J. (1983). Compton effect - a classical treatment. European Journal of Physics, Vol.4, (November, 1983), pp.205-213 
DuMond, J. (1933). The Linear Momenta of Electrons in Atoms and in Solid Bodies as Revealed by X-Ray Scattering. Review of Modern Physics, Vol.5, No.1, (January 1933), pp. 1-33

Eve, A. (1904). On the secondary radiation caused by the beta and gamma rays of radium. Philosophical Magazine, Vol.8, No.48, (December, 1904), pp. 669-685

Feynman, R. (1965). The Feynman Lectures on Physics, Addison-Wesley, ISBN 0-8053-9045-6, New York, USA

Florance, D. (1910). CIV. Primary and secondary yrays. Philosophical Magazine, Vol.20, No.120, (December 1910), pp. 921-938

Glauber, R. (2006). Nobel Lecture: One hundred years of light quanta. Review of Modern Physics, Vol.78, No.4, (November 2006), pp. 1267-1278

Gray, J. (1920). The scattering of X- and y-rays. Journal of The Franklin Institute, Vol.190, No.5, pp. 633-655

Heisenberg, W. (1958). Physics and Philosophy: The Revolution in Modern Science, Harper \& Brothers, ISBN: 0061305499, New York, USA

Jauncey, G. (1925) Quantum Theory of the Unmodified Spectrum Line in the Compton Effect, Physical Review, Vol.25,No.3, (March 1925), pp. 314-321

Kidd, R., Ardini, J. \& Anton, A. (1989) Evolution of the modern photon. American Journal of Physics, Vol.57, No.1,(January, 1989), pp.27-34

Kim, S. (1993) Principle of Random Wave-Function Phase of the Final State in Free-Electron Emission in Wiggler. APEIRON, Vol.17, pp.13-17

Klein, O. \& Nishina, Y (1929). Über die Streuung von Strahlung durch freie Elektronen nach der neuen relativistischen Quantendynamik von Dirac. Zeitschrift für Physik, Vol.52, No.11, pp. 853-868

Lorentz, H. (1916). The Theory of Electrons, Teubner, Leipzig Lamb W. \& Scully, M. (1969). The Photoelectric Effect Without Photons, In: Polarization, Matter and Radiation, Presses Universitaires de France, pp.363-369, Paris

Mie, C. (1908). Beiträge zur Optik trüber Medien, speziell kolloidaler Metallösungen. Annalen der Physik, Vol.25, No.3, pp. 377-445

Pimpale, A. \& Mande, Ch. (1984). Raman effect in x-ray region. Pramana, A Journal of Physics, Vol.23, No.3, (September 1984), pp.279-295.

Raman, G. (1928) A new radiation. Indian Journal of Physics, Vol.21, (March 1928), pp.368-377

Ross P. \& Kirkpatrick P. (1934). The Constant in the Compton Equation. Physical Review, Vol.45, No.3, (February 1934), pp. 223-223

Schrödinger, E. (1926). Quantizierung als Eigenwertproblem (Erste Mitteilung). Part I. Annalen der Physik, Vol.79, No.4, pp. 361-376

Schrödinger, E. (1927). Uber den Comptoneffekt. Annalen der Physik, Vol.82, No.4, (January 1927), pp. 257-264

Smith, L. \& Purcell, E. (1953). Visible Light from Localized Surface Charges Moving across a Grating, Physical Review, Vol.92, No.4, (November 1953), pp. 1069-1069

Stuewer, R. (1975). The Compton effect: turning point in physics, Science History Publications, ISBN 9780882020129, New York, USA

Styer, D., Balkin, M., Becker, K., Burns, M., Dudley, C., Forth, S., Gaumer, J., Kraer, M., Oertel, D., Park, L., Rinkoski, M., Smith, C. \& Wotherspoon, D. (2002). Nine formulations of quantum mechanics. American Journal of Physics, Vol.73, No.3, (November 2001), pp.288-297 
Tamm, I. (1930). Uber die Wechselwirkung der freien Elektronen mit der Strahlung nach der Diracschen Theorie des Elektrons und nach der Quantenelektrodynamik. Zeitschrift fur Physik, Vol.62, No.7, pp.545-568

Wheeler, J. \& Feynman, R. (1945). Interaction with the Absorber as the Mechanism of Radiation. Review of Modern Physics, Vol.17, No.2,3, (April 1945)

Wolf, M. (n. d.). Beyond the Point Particle - a Wave Structure for the Electron, In: Galilean Electrodynamics 6, No. 5, (May1998), < http://mwolff.tripod.com/point.html > 


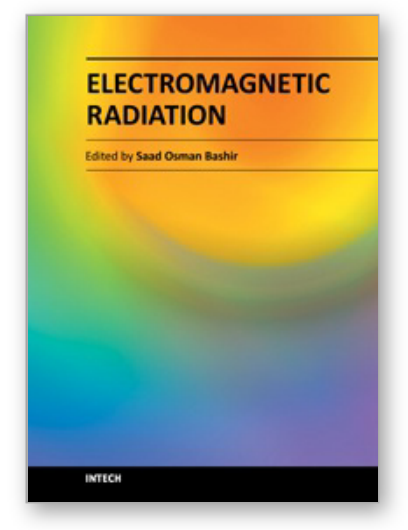

\author{
Electromagnetic Radiation \\ Edited by Prof. S. O. Bashir
}

ISBN 978-953-51-0639-5

Hard cover, 288 pages

Publisher InTech

Published online 05, June, 2012

Published in print edition June, 2012

The application of electromagnetic radiation in modern life is one of the most developing technologies. In this timely book, the authors comprehensively treat two integrated aspects of electromagnetic radiation, theory and application. It covers a wide scope of practical topics, including medical treatment, telecommunication systems, and radiation effects. The book sections have clear presentation, some state of the art examples, which makes this book an indispensable reference book for electromagnetic radiation applications.

\title{
How to reference
}

In order to correctly reference this scholarly work, feel free to copy and paste the following:

V.V. Aristov (2012). Neoclassical Theory of X-Ray Scattering by Electrons, Electromagnetic Radiation, Prof. S. O. Bashir (Ed.), ISBN: 978-953-51-0639-5, InTech, Available from: http://www.intechopen.com/books/electromagnetic-radiation/neoclassical-theory-of-x-ray-scattering-byelectrons

\section{INTECH}

open science | open minds

\section{InTech Europe}

University Campus STeP Ri

Slavka Krautzeka 83/A

51000 Rijeka, Croatia

Phone: +385 (51) 770447

Fax: +385 (51) 686166

www.intechopen.com

\section{InTech China}

Unit 405, Office Block, Hotel Equatorial Shanghai

No.65, Yan An Road (West), Shanghai, 200040, China

中国上海市延安西路65号上海国际贵都大饭店办公楼 405 单元

Phone: +86-21-62489820

Fax: +86-21-62489821 
(C) 2012 The Author(s). Licensee IntechOpen. This is an open access article distributed under the terms of the Creative Commons Attribution 3.0 License, which permits unrestricted use, distribution, and reproduction in any medium, provided the original work is properly cited. 